\title{
COLLINEATION GROUPS PRESERVING AN OVAL IN A PROJECTIVE PLANE OF ODD ORDER
}

\author{
MAURO BILIOTTI and GABOR KORCHMAROS
}

(Received 15 February 1988)

\begin{abstract}
In this paper we investigate the structure of a collineation group $G$ of a finite projective plane $\Pi$ of odd order, assuming that $G$ leaves invariant an oval $\Omega$ of $\Pi$. We show that if $G$ is nonabelian simple, then $G \cong P S L(2, q)$ for $q$ odd. Several results about the structure and the action of $G$ are also obtained under the assumptions that $n \equiv 1(4)$ and $G$ is transitive on the points of $\Omega$.
\end{abstract}

1980 Mathematics subject classification (Amer. Math. Soc.) (1985 Revision): primary 51 E 15, 51 A 10; secondary 20 B 25.

\section{Introduction}

In a previous work [2] the authors consider a finite projective plane $\Pi$ of odd order, endowed with a collineation group $G$ which preserves an oval $\Omega$ and acts primitively on its points. In this case $\Pi$ is Desarguesian of order $q, \Omega$ is a conic and $G \cong P S L(2, q)$, with only one exception.

When one attempts to study the same problem under the weaker assumption that $G$ is transitive on $\Omega$, the situation appears to be much more involved. Indeed, $G$ may be solvable, fixing a point-line pair (actually, this happens in the Desarguesian plane) and it seems very difficult to determine the action of $G$ on the whole plane.

On the other hand, for such an investigation it is essential to know which simple groups can preserve an oval in a projective plane of odd order. Of course, this subject may be interesting in itself. Here, we give a complete answer to the question by showing the following theorem.

This research was supported in part by a grant from the $M$. P. I.

(C) 1990 Australian Mathematical Society 0263-6115/90\$A2.00+0.00 
THeOREM A. Let $G$ be a collineation group of a projective plane $\Pi$ of odd order. If $G$ is nonabelian simple and leaves invariant an oval of $\Pi$, then $G \cong P S L(2, q)$ for $q$ odd and all involutions in $G$ are homologies.

The proof does not need the classification of simple groups. Indeed the authors [2] already proved that a group preserving an oval in a projective plane of odd order must have 2-rank at most 3.

In Section 4 we deal with the case of a projective plane of order $n \equiv 1$ (4) and among other things, we prove the following theorem.

THEOREM B. Let $G$ be a collineation group of a projective plane $\Pi$ of order $n \equiv 1$ (4) such that $G$ preserves an oval $\Omega$, acts transitively on its points and does not contain any nontrivial subgroup with the same properties. Then one of the following holds.

(i) $G=O(G) \cdot\langle\tau\rangle$, where $\tau$ is a suitable 2-element of $G$.

(ii) $\Pi$ has order $q(q+1)-1$ or $q(q-1)-1$ according to whether $G \cong$ $\operatorname{PSL}(2, q)$ with $q \equiv 1$ (4) or $G \cong \operatorname{PSL}(2, q)$ with $q \equiv 3$ (4) and there is exactly one homology axis through each point of $\Omega$.

(iii) $\Pi$ is a desarguesian plane, $\Omega$ is a conic and either

(a) $\Pi$ has order $q$ with $q \neq 5,9$ and 29 , and $G$ acts on $\Omega$ as $\operatorname{PSL}(2, q)$ in its usual doubly transitive representation, or

(b) $\Pi$ has order 9 and $G$ acts on $\Omega$ as $P S L(2,5)$ in its primitive representation of degree 10 .

All situations (i)-(iii) actually occur in a Desarguesian plane. When $\Pi$ is Desarguesian of order 5 or 29 , the case (ii) occurs for $G \cong P S L(2,3) \cong A_{4}$ and $G \cong P S L(2,5)$, respectively.

The analogous problem in a plane of order $n \equiv 3$ (4) seems to be very difficult. For similar investigations in a projective plane of even order see [1] and [3].

\section{Preliminary results}

We shall use standard notation. The necessary background about projective planes may be found in [5].

Let $\Pi$ be a projective plane of odd order $n$. If $H$ is a collineation group of $\Pi$, then $F(H)$ denotes the substructure of $\Pi$ consisting of those points and lines which are fixed by each element of $H$. We shall write $F(\alpha)$ instead of $F(\langle\alpha\rangle)$ for any $\alpha \in H$. A triangular group $K$ of homologies of $\Pi$ is a Klein 
group of collineations of $\Pi$ whose involutions are homologies and such that $F(K)$ is a triangle.

We also recall that, following Hering [10], a collineation group $G$ of $\Pi$ is called irreducible if $G$ does not fix any point, line or triangle of $\Pi$ and strongly irreducible if $G$ is irreducible and does not leave invariant any proper subplane of $\Pi$.

For what concerns finite groups the reader is referred to [8] and [11]. For simple groups see also [9]. In particular, the classification of the subgroups of $\operatorname{PSL}(2, q)$ is assumed to be known (see [11, II, Hauptsatz 8.27]). Here we only recall that a group $G$ is said to be of 2-rank $h$ if $h$ is the maximum rank of an abelian 2-subgroup of $G$.

Simple groups of 2-rank at most 3 are well known.

RESULT 2.1. Let $G$ be a finite, nonabelian, simple group of 2-rank at most 3. Then either

(i) $G \cong G_{2}(q),{ }^{3} D_{4}(q),{ }^{2} G_{2}\left(3^{n}\right), P S L(2,8), P S U\left(3,8^{2}\right), S z(8), M_{12}, J_{1}$, $O N$, where $q$ and $n$ are odd, $n>1$, and $G$ has 2-rank 3, or

(ii) $G \cong P S L(2, q)(q \geq 5), \operatorname{PSL}(3, q), \operatorname{PSU}\left(3, q^{2}\right), \operatorname{PSU}\left(3,4^{2}\right), A_{7}, M_{11}$, where $q$ is odd, and $G$ has 2-rank 2.

Moreover, $G$ has only one class of involutions except when $G \cong M_{12}$, in which case $G$ has two classes of involutions.

Proof. See [9, Theorems 1.86 and 2.168], for assertions (i) and (ii). The number of involution classes is well-known (for example, see [9] for $G_{2}(q)$ and ${ }^{3} D_{4}(q)$, [12] for ${ }^{2} G_{2}\left(3^{n}\right)$ and $S z(8)$, [15] for sporadic simple groups).

Now, we enumerate some known results about the ovals of $\Pi$.

An oval $\Omega$ of $\Pi$ is a set of $n+1$ points of $\Pi$, no three of which are collinear. A line $l$ of $\Pi$ is called an external line, a tangent or a secant of $\Omega$ according to whether $|l \cap \Omega|=0,1$ or 2 . Each point of $\Pi$, not in $\Omega$, lies in either two or no tangents of $\Omega$ and it is called an external point or an internal point, respectively. For more details see [5].

Let $G$ be a collineation group of $\Pi$ which leaves $\Omega$ invariant.

Result 2.2. The following hold.

(1) Any perspectivity in $G$ is an involutory homology and two distinct homologies in $G$ have both distinct centres and distinct axes.

(2) If $K$ is a Klein subgroup of $G$, then $K$ contains an involutory homology which induces an even permutation on $\Omega$.

(3) $G$ does not contain any elementary abelian subgroup of order 8 generated by three homologies.

(4) G has 2-rank at most 3. 
For a proof see [2, Propositions 2.1, 2.3 and 2.4].

RESULt 2.3. If $\sigma$ is an involution of $G$, then one of the following holds.

(1) $\sigma$ induces an odd permutation on $\Omega$. Moreover, (a) if $\sigma$ is a $(L, l)$-homology, then $L$ is an internal point and $l$ is an external line of $\Omega$, or $L$ is an external point and $l$ is a secant of $\Omega$, according to whether $n \equiv 1$ (4) or $n \equiv 3$ (4),

(b) if $\sigma$ is a Baer collineation, then either $\Omega \cap F(\sigma)=\varnothing$, or $\Omega \cap F(\sigma)$ is an oval of $F(\sigma)$ and $\sqrt{n} \equiv 3(4)$.

(2) $\sigma$ induces an even permutation on $\Omega$. Moreover,

(a) if $\sigma$ is a $(L, l)$-homology, then $L$ is an internal point and $l$ is an external line of $\Omega$, according to whether $n \equiv 1$ (4) or $n \equiv 3$ (4).

(b) if $\sigma$ is a Baer collineation, then $\Omega \cap F(\sigma)$ is an oval of $F(\sigma)$.

In addition, when $\sigma$ is a Baer collineation, the following hold:

(i) if $\Omega \cap F(\sigma)=\varnothing$, then $(n+1) / 2$ lines of $F(\sigma)$ are secants of $\Omega$, while the remaining $(\sqrt{n}+1)^{2} / 2$ lines are external to $\Omega$;

(ii) if $\Omega \cap F(\sigma)$ is an oval of $F(\sigma)$, then no line of $F(\sigma)$ is external to $\Omega$.

For a proof see [2, Proposition 2.2].

LEMMA 2.4. If $G$ is a Klein group and all involutions in $G$ are homologies, then $G$ acts f.p.f. on $\Omega$.

Proof. $F(G)$ is a triangle, by Result 2.2(1), and each of its vertices is the centre of an involutory homology. So, a vertex of $F(G)$ cannot lie in $\Omega$ by Result 2.3, (1.a) and (2.a).

\section{Simple groups preserving an oval}

A collineation group leaving an oval invariant possesses the following remarkable "local" property.

Proposition 3.1. Let $G$ be a collineation group of $\Pi$ leaving invariant an oval $\Omega$. Suppose that $G$ contains a Klein group $K$ and let $S$ be the subgroup of $G$ generated by all involutions lying in $\mathscr{N}_{G}(K)$. Then either $S$ centralizes an involution in $K$ or $S \cong S_{4}$.

Proof. Suppose that $S$ does not centralize any involution in $K$. Then all involutions in $K$ are conjugate and hence $K$ is a triangular group of homologies by Result 2.2, (1) and (2). 
Let $P, Q, R$ be the vertices of $F(K)$ and let $p, q, r$ be their opposite sides. $\mathscr{N}_{G}(K)$ permutes cyclically the sides of $F(K)$ so that either all sides of $F(K)$ are secants of $\Omega$ or all sides of $F(K)$ are external to $\Omega$, according to Result 2.3. The proof develops into the following steps.

(1) If $\alpha$ and $\beta$ are two distinct involutory homologies in $\mathscr{N}_{G}(K)-K$, then either $|\alpha \beta|=2$ or $|\alpha \beta|=3$ according to whether the centres of $\alpha$ and $\beta$ lie in the same side or in two distinct sides of $F(K)$.

Let $A$ and $B$ be the centres of $\alpha$ and $\beta$ respectively.

Suppose that $A$ and $B$ lie in the same side, say $p$, of $F(K)$. Since both $\alpha$ and $\beta$ interchange the vertices $Q$ and $R$ and their axes pass through $P, \alpha \beta$ fixes $F(K)$ pointwise. By [5, Proposition 4.2.6], $\langle\alpha \beta\rangle$ acts semiregularly on the points of $\Pi$ different from $P$ and not lying in $p$. So, $\langle\alpha \beta\rangle$ acts semiregularly on the points of $r$ different from $P$ and $Q$ and hence $|\alpha \beta| \mid n-1$. If $r$ is a secant of $\Omega, r \cap \Omega$ is an orbit of length 2 of $\langle\alpha \beta\rangle$ which is distinct from $\{P, Q\}$ by Lemma 2.4. Therefore, $|\alpha \beta|=2$. If $r$ is an external line of $\Omega$ then each side of $F(K)$ is external to $\Omega$. Therefore, $\langle\alpha \beta\rangle$ acts semiregularly on $\Omega$ and hence $\mid \alpha \beta \| n+1$. We then have that $|\alpha \beta|=2$ since $|\alpha \beta| \mid n-1$, as we already showed.

Now, suppose that $A$ and $B$ lie in two distinct sides, say $p$ and $q$, of $\Omega$. Both $\alpha \beta \alpha$ and $\beta \alpha \beta$ are involutory homologies in $\mathscr{N}_{G}(K)-K$ whose centres lie in the line $A B$. Since these centres must lie in the third side $r$ of $\Omega$, they both coincide with the point $r \cap A B$. Thus, $\alpha \beta \alpha=\beta \alpha \beta$ by Result 2.2(1) and hence $|\alpha \beta|=3$.

(2) $\mathscr{N}_{G}(K)-K$ contains exactly six involutory homologies.

Now $\mathscr{N}_{G}(K)-K$ must contain some involutory homology since otherwise $S=K$, contrary to our assumption that no involution in $K$ is centralized by $S$. Let $\alpha$ be an involutory homology in $\mathscr{N}_{G}(K)-K$ whose centre $A$ lies in $p$. The side $p$ must contain the centre of another involutory homology $\beta \in \mathscr{N}_{G}(K)-K$ since the involutory $(Q, q)$-homology does not fix $A$. Suppose that $p$ contains the centre $C$ of a third involutory homology $\gamma \in \mathscr{N}_{G}(K)-K$, different from $\alpha$ and $\beta$. Then $\langle\alpha, \beta, \gamma\rangle$ is an elementary abelian group of order 4 by (1) and Result 2.2 (3). Nevertheless, $\langle\alpha, \beta, \gamma\rangle$ centralizes the involutory $(P, p)$-homology $\nu$ since it fixes the axis of $\nu$. This contradicts Result 2.2 (3) since $\langle\alpha, \beta, \gamma, \nu\rangle$ would be an elementary abelian group of order 8 . Since $\mathscr{N}_{G}(K)$ is transitive on the sides of $F(K)$ our assertion is proved.

Using (1) and (2) it is easily seen that the set $D$ consisting of the six involutions in $\mathscr{N}_{G}(K)-K$ verifies the following conditions:

(a) all involutions in $D$ are conjugate;

(b) $|\alpha \beta|=2$ or 3 for each $\alpha, \beta \in D$;

(c) three pairwise different elements of $D$ never generate a 2-group;

(d) $C_{D}(\alpha) \neq \alpha$ for each $\alpha \in D$. 
By a result of Fisher [6], $\langle D\rangle \cong S_{4}$. It is easily seen that $\langle D\rangle$ contains $K$ and hence $S \cong S_{4}$.

Throughout the rest of this section we assume that $G$ is a nonabelian simple group which leaves invariant an oval $\Omega$ of $\Pi$.

Proposition 3.2. $G$ is an irreducible collineation group of $\Pi$.

Proof. $G$ contains a triangular group $K$ of homologies by Result 2.2 (2) and by [7, Corollary 1]. Hence, $G$ cannot fix a point $P \in \Omega$ by Lemma 2.4 .

Suppose that $G$ fixes a point $P \notin \Omega$. The group $K$ must contain an homology $\sigma$ with the centre $P$. Since $\sigma$ is the unique homology with that centre, it is central in $G$. This is a contradiction, being $G$ simple.

So, $G$ does not fix any point and, dually, $G$ does not fix any line.

If $G$ leaves invariant a triangle $\Delta$, then $G$ must fix $\Delta$ pointwise because $G$ is simple. This has been already excluded.

Lemma 3.3. The cases $G \cong P S U\left(3, q^{2}\right)$ or $A_{7}$ cannot occur.

Proof. All involutions in $G$ are homologies by Results 2.1 and 2.2(2). Let $K$ be a Klein subgroup of $G$.

Suppose that $G \cong P S U\left(3, q^{2}\right)$. The group $S$ generated by all involutions in $\mathscr{N}_{G}(K)$ has order $6(q+1)^{2} / d$, where $d=(3, q+1)$, and is transitive on the vertices (the sides) of $F(K)$ (see [14, Theorem 3]). By Proposition 3.1 we should have $S \cong S_{4}$. So, we have a contradiction.

Now suppose that $G \cong A_{7}$. In this case the group $S$ is of type $\left(E_{4} \times Z_{3}\right) S_{3}$ and again, it is transitive on the vertices (the sides) of $F(K)$ (see [4, Appendix 3] and also recall that $A_{7} \leq P S U\left(3,5^{2}\right)$ ). This contradicts Proposition 3.1.

LEMmA 3.4. The cases $G \cong P S L(3, q), M_{11}$ or $M_{12}$ cannot occur.

Proof. Again, $G$ contains involutory homologies by Result 2.2(2).

Let $G \cong P S L(3, q)$. By [13, Theorem C], $G$ contains non-trivial elations, contrary to Result $2.2(1)$.

The group $G$ is strongly irreducible on the subplane of $\Pi$ generated by the centres and the axes of involutory homologies in $G$ (see [10, Lemmas 3.3 and 3.5]). So, $G \not M_{11}$ or $M_{12}$ by [15].

We notice that the same technique of Lemma 3.3 could be essentially used also in the proof of Lemma 3.4.

Now, we can prove Theorem $A$.

Proof of Theorem A. Suppose that $n \equiv 3$ (4). 
A 2-group of collineations of $\Pi$ is cyclic, dihedral, semidihedral, or a generalized quaternion group (see [5, Proposition 4.2.4]). As $G$ is simple, a Sylow 2-subgroup of $G$ is dihedral or semidihedral (see [9, Section 1]). By the Gorenstein-Walter and Alperin-Brauer-Gorenstein theorems [9] we then have that

$G \cong P S L(2, q)$ ( $q$ odd, $q \geq 5), P S L(3, q)$ (for $q \equiv 3(4)$ ),

$P S U\left(3, q^{2}\right)($ for $q \equiv 1(4)), A_{7}$ or $M_{11}$.

Suppose that $n \equiv 1(4)$.

By Result 2.2(3) and (4), either $G$ has 2-rank 2 or $G$ has 2-rank 3 and possesses more than one class of involutions. By Result 2.1 we then have that

$$
\begin{aligned}
& G \cong \operatorname{PSL}(2, q)(q \geq 5), \operatorname{PSL}(3, q), \operatorname{PSU}\left(3, q^{2}\right), \operatorname{PSU}\left(3,4^{2}\right), A_{7}, \\
& M_{11}, \text { where } q \text { is odd, or } G \cong M_{12} .
\end{aligned}
$$

By Lemmas 3.3 and 3.4 we have to consider only the case $G \cong P S U\left(3,4^{2}\right)$. Note that the homology axes in $G$ are secants of $\Omega$ by Result 2.3(2.a). Let $P$ be any point of $\Omega$ which is fixed by the involutory homology $\sigma . C_{G}(\sigma)$ contains a subgroup $C$ of index two fixing $P$. By Lemma 2.4, the group $C$ cannot contain any involution different from $\sigma$ and commuting with $\sigma$. Nevertheless, in the case under consideration, a Sylow 2-subgroup $T$ of $G$ contains a Klein group $K$ such that each element in $K$ is a square in $T$ (see for example [12]). So, we have a contradiction. This completes the proof.

\section{Transitive groups}

The study of collineation groups acting transitively on the points of an invariant oval seems to be rather difficult. Here we give only some partial results.

Throughout this section we suppose that the plane $\Pi$ has order $n \equiv 1$ (4).

LEMMA 4.1. Let $P$ be a p-group of collineations of $\Pi$ which preserves an oval $\Omega$. If $p$ is odd and $P$ acts f.p.f. on $\Omega$ then one of the following holds

(j) $F(P)=\{L, l\}$, where $L$ is an internal point and $l$ an external line of $\Omega$.

(ji) $F(P)$ is a subplane whose points are internal to $\Omega$ and whose lines are external to $\Omega$.

Furthermore, if $\sigma$ is an involutory collineation of $\Pi$ which preserves $\Omega$, induces an even permutation on $\Omega$ and normalizes $P$, then $\sigma$ is a homology and $F(P)$ is as in (j). 
Proof. Clearly, $P$ can fix only internal points and external lines of $\Omega$ since it acts f.p.f. on $\Omega$. Furthermore, $p \mid n+1$. So, $(p, n(n-1) / 2)=1$ and hence $P$ fixes at least one internal point $L$ and one external line $l$ of $\Omega$. Suppose that $P$ fixes an external line $m$ of $\Omega$ different from $l$. Then $P$ fixes $l \cap m$. Futhermore, $P$ fixes at least two other points both on $l$ and $m$, being $(p, n)=(p, n-1)=1$. Hence, $F(P)$ is a subplane. The same holds if $P$ fixes an internal point of $\Omega$ different from $L$.

We have to prove the last assertion. Assume that $\sigma$ satisfies the required assumptions. Then $\sigma$ must fix some line $h$ in $F(P)$. If $\sigma$ were a Baer collineation then $h$ should be a secant or a tangent of $\Omega$ by Result $2.3(2 \mathrm{~b})$ and (ii). This is impossible. Therefore, $\sigma$ is a homology. When $F(P)$ is a subplane the axis $m$ of $\sigma$ lies in $F(P)$. Since $m$ is a secant of $\Omega$ by Result 2.3(2.a), this situation cannot occur.

Now we can prove the following

Proposition 4.2. Let $G$ be a collineation group of $\Pi$ which preserves an oval $\Omega$ and acts transitively on its points. If $G_{0}=G \cap$ Alt $\Omega$ has even order, then $G_{0}$ contains involutory homologies.

Proof. Suppose that all involutions in $G_{0}$ are Baer collineations. By [2, Proposition 2.5], a Sylow 2-subgroup $S$ of $G_{0}$ is cyclic. Thus, $G_{0}=O\left(G_{0}\right) \cdot S$ by the well-known result of Burnside (for example see [11, IV, Satz, 2.8]).

Since $O\left(G_{0}\right) \unlhd G$, there exists a minimal normal subgroup $M$ of odd order in $G$. As $G$ is transitive on $\Omega, M$ acts f.p.f. on $\Omega$. Let $\sigma$ be an involution in $G_{0}$. By Lemma 4.1, $\sigma$ should be a homology and we have a contradiction.

Proposition 4.2 allow us to use Hering's theory [10] in studying collineation groups $G$ which are transitive on an oval and irreducible over the plane provided that $4 \| G \mid$. So, one of the main problems is to decide when $G$ is irreducible. Here we put forward the following conjecture.

Conjecture. A collineation group which preserves an oval $\Omega$ in a projective plane of odd order and acts transitively on the points of $\Omega$ is irreducible if and only if it is nonsolvable.

In the following, we give some results which seem to confirm this conjecture.

Proposition 4.3. Let $G$ be a collineation group of $\Pi$ which preserves an oval $\Omega$ and acts transitively on its points. If $G$ is not irreducible over $\Pi$ and 4||$G \mid$, then one of the following holds.

(1) $G$ fuxes a point-line pair $P, l$ of $\Pi$. Furthermore,

(a) the Sylow 2-subgroups of $G_{0}=G \cap \mathrm{Alt} \Omega$ are cyclic or generalized quaternion groups. 
(b) the group $O(G) \cdot\langle\tau\rangle$, where $\tau$ is a suitable 2-element of $G$, is transitive on $\Omega$.

(2) $G$ leaves invariant a triangle, $\Pi$ has order 5 and $G \cong A_{4}$ or $S_{4}$.

Furthermore, all involutions in $G_{0}$ are homologies.

Proof. Suppose that $G$ fixes a point $P$.

If $P$ is an external point of $\Omega$ then $G$ either fixes or interchanges the tangents at $\Omega$ through $P$. This is in contrast with the transitivity of $G$ on $\Omega$. So, $P$ is an internal point of $\Omega$.

An involution $\sigma \in G_{0}$ cannot be a Baer collineation since it fixes the point $P$ which is internal to $\Omega$. Likewise, $\sigma$ cannot be an homology with the centre $P$ (see Result 2.3). Therefore, all involutions in $G_{0}$ are homologies whose axes pass through $P$ and any two of them must generate a group of odd order. This proves (1.a). Furthermore, by a result of Glauberman (see [7, Corollary 5.3]), the involutions in $G_{0}$ generate a group $H$ such that $[H: O(H)]=2$. Since $G$ is transitive on $\Omega$, there is exactly one homology axis through each point of $\Omega$ and the homology axes lie in the same orbit under $O(H)$. So, $O(H)$ splits the points of $\Omega$ into two orbits of length $(n+1) / 2$. Furthermore, there must be an element $\tau$ of $G$ having order a power of two and interchanging the orbits of $O(H)$. Since $O(H) \leq O(G)$, we have (1.b).

The group $G$ possesses a minimal normal subgroup $M$ of odd order which acts f.p.f. on $\Omega$ since $G$ is transitive on $\Omega$. Furthermore, $G$ contains involutory homologies inducing even permutations on $\Omega$. Therefore, $F(M)=\{P, l\}$ for an external line $l$ of $\Omega$ by Lemma 4.1. We must have $F(G)=F(M)$ and (1) is proved.

Dually, the same conclusion holds when $G$ fixes a line.

Suppose that $G$ leaves invariant a triangle $\Delta$.

We may assume that $G$ does not fix any element of $\Delta$ and that none of the sides of $\Delta$ is a tangent of $\Omega$. If the sides of $\Delta$ are secants of $\Omega$ then $\Pi$ has order 5 in view of the transitivity of $G$ on $\Omega$. Thus, $\Pi$ is Desarguesian and the oval $\Omega$ is left invariant by the group $\operatorname{PGL}(2,5)$ which contains exactly the subgroups $A_{4}$ and $S_{4}$ acting on the points of $\Omega$ in the required manner.

If the sides of $\Delta$ are external to $\Omega$, let $G_{0}(\Delta)$ be the subgroup of $G_{0}$ fixing $\Delta$ pointwise. An involution in $G_{0}$ cannot be a Baer collineation since it fixes external lines of $\Omega$. An involution in $G_{0}(\Delta)$ cannot be an homology since its axis should be one of the sides of $\Delta$ which are external to $\Omega$ (see Result 2.3). Therefore, $G_{0}(\Delta)$ has odd order and $G_{0} / G_{0}(\Delta) \leq S_{3}$. If $G_{0}(\Delta) \neq\langle 1\rangle$, $G$ contains a minimal normal subgroup of odd order and hence, by Lemma 4.1, $F(G)$ consists of a point-line pair, contrary to our assumption. The case 
$G_{0}(\Delta)=\langle 1\rangle$ and $G_{0} \cong S_{3}$ may be excluded in the same manner. So, the case under consideration cannot occur.

When $G$ is solvable, $G$ cannot be irreducible over $\Pi$. Namely, we have

Proposition 4.4. Let $G$ be a collineation group of $\Pi$ which preserves an oval $\Omega$ and acts transitively on its points. If $G$ is solvable and $4 \| G \mid$, then either the case (1) or the case (2) of Proposition 4.3 occurs and all involutions in $G_{0}$ are homologies.

Proof. Suppose that $G$ possesses a minimal normal subgroup $M$ of odd order. $M$ acts f.p.f. on $\Omega$ because $G$ is transitive on $\Omega$. By Lemma 4.1, the involutions in $G_{0}$ are homologies and $F(M)=\{L, l\}$, where $L$ is an internal point and $l$ an external line of $\Omega$. We must have $F(G)=F(M)$ and hence the case (1) of Proposition 4.3 occurs.

Now, assume that every minimal normal subgroup of $G$ has even order. Since $G_{0} \unlhd G$, there exists a minimal normal subgroup $M$ of $G$ contained in $G_{0}$.

The case $|M|=2$ cannot occur. Indeed, the unique involution in $M$ would fix some point on $\Omega$, contrary to the transitivity assumption. So, $|M|=4$ and $M$ is a triangular group of homologies as easily follows from Result 2.2. Thus, $G$ leaves invariant $F(M)$ and we have the case (2) of Proposition 4.3.

For "minimal" transitive groups we have the following

COROLlaRY 4.5. Let $G$ be a collineation group of $\Pi$ which preserves an oval $\Omega$, acts transitively on its points and does not contain any nontrivial subgroup with the same properties. If $4 \| G \mid$, then $G$ is solvable if and only if it is not irreducible over $\Pi$.

Proof. If $G$ is solvable then $G$ cannot be irreducible by Proposition 4.4. Suppose that $G$ is not irreducible. By Proposition 4.3, either $G=O(G) \cdot\langle\tau\rangle$, where $\tau$ is an element of $G$ of order $2^{r}$, with $r \geq 2$, or $G_{0}=G \cap$ Alt $\Omega=$ $O(G) \cdot\langle\sigma\rangle$ for an homology $\sigma$ and $G=G_{0} \cdot\langle\tau\rangle$ for a suitable element of order 2 of $G$. So, $G$ is solvable.

Another corollary to Proposition 4.3 is the following

COROLlary 4.6. With the same assumptions of Proposition 4.3 suppose that the case (1) holds for $G$. If none of the elements of odd order of $G$ is planar, then $G_{0}=G \cap$ Alt $\Omega$ is a Frobenius group. 
Proof. We may regard $G_{0}$ as acting on the pencil $\Xi$ consisting of the secants of $\Omega$ through the fixed point $P$. Clearly, $G_{0}$ is transitive on $\Xi$. Furthermore, a nontrivial element of odd order of $G_{0}$ cannot fix two distinct lines of $\Xi$. Indeed, it should fix at least four points on $\Omega$ and hence it would be planar.

Suppose that a centre $A$ of an involutory homology $\alpha \in G_{0}$ lies on a line $b$ of $\Xi$. Then $\alpha$ centralizes the unique involutory homology of $G_{0}$ with the axis $b$. This contradicts Proposition 4.3 (1.a). So, an involutory homology in $G_{0}$ fixes exactly one line of $\Xi$. It follows that an element of even order of $G_{0}$ cannot fix two distinct lines of $\Xi$.

Therefore $G_{0}$ acts on $\Xi$ as a Frobenius group.

The rest of this section is devoted to the study of those groups which are transitive on an oval and irreducible over the plane.

LEMMA 4.7. Let $G$ be a collineation group of $\Pi$ which preserves an oval $\Omega$ and acts transitively on its points. If $G$ is irreducible over $\Pi$ and $4 \| G \mid$, then one of the following holds:

(i) $M \leq G \leq \operatorname{Aut}(M)$;

(ii) $G=G_{0} \times K$, where $G_{0}=G \cap$ Alt $\Omega, K$ has order 2 and $M \leq G_{0} \leq$ $\operatorname{Aut}(M)$;

for a suitable nonabelian simple group $M$.

Proof. $G$ contains involutory homologies by Proposition 4.2. By [10, Lemmas 3.3 and 5.3], the centres and the axes of homologies in $G$ generate a subplane $\Sigma$ of $\Pi$ and $G$ is strongly irreducible over $\Sigma$. Suppose that $\Sigma=$ $\Pi$. By [10, Theorem 5.5], either we have (i) or the unique minimal normal subgroup $M$ of $G$ is elementary abelian of order 9 and $G / M \leq \operatorname{Aut}(M)$. The latter possibility cannot occur since $G$ would be solvable, in contrast with Proposition 4.4.

Now, suppose that $\Sigma \neq \Pi$. Then the following hold.

(1) $\Omega \cap \Sigma=\varnothing$.

(2) There is exactly one line of $\Sigma$ through each point of $\Omega$ and this line is the axis of an involutory homology.

(3) The kernel $K$ of the representation of $G$ on $\Sigma$ has order at most 2 .

(1) follows from the transitivity of $G$ on $\Omega$.

(2) $G_{0}$ contains involutory homologies whose axes are secants of $\Omega$ by Proposition 4.2. By (1) and the transitivity of $G$ on $\Omega$ we have (2).

(3) Suppose that $K \neq\langle 1\rangle$. Using (2) it is easily seen that an element of odd order of $K$ fixes $\Omega$ pointwise and hence it is the identity. So, $|K|=2^{h}$ and $K$ is a cyclic group by [2, Proposition 2.5]. The unique involution $\sigma$ in 
$K$ cannot fix any point on $\Omega$ since it is central in $G$. Therefore, $\sigma$ induces an odd permutation on $\Omega$ and hence $|K|=2$.

When $K=\langle 1\rangle$ we have (i) by [10, Theorem 5.5]. Otherwise, $G=G_{0} \times K$. The group $G_{0}$ is faithful and strongly irreducible over $\Sigma$ since $G$ and $G_{0}$ act on $\Sigma$ in the same manner. Thus, $M \leq G_{0} \leq \operatorname{Aut}(M)$ for a nonabelian simple group $M$, again by [10, Theorem 5.5] and by Proposition 4.4.

Now, we make use of Theorem A to obtain the following result.

THEOREM 4.8. Let $G$ be a collineation group of $\Pi$ which preserves an oval $\Omega$ and acts transitively on its points. If $G$ is irreducible over $\Pi$ and $4 \| G \mid$, then $G$ is strongly irreducible over $\Pi$ and $M \leq G \leq \operatorname{Aut}(M)$, where $M \cong P S L(2, q)$. Furthermore, $M$ is transitive on the points of $\Omega$ and one of the following holds:

(1) $\Pi$ has order $q(q+1)-1$ or $q(q-1)-1$ according to whether $M \cong$ $P S L(2, q)$ with $q \equiv 1$ (4) or $M \cong P S L(2, q)$ with $q \equiv 3$ (4) and there is exactly one homology axis through each point of $\Omega$.

(2) $\Pi$ is a Desarguesian plane, $\Omega$ is a conic and either

(a) $\Pi$ has order $q$ and $M$ acts on $\Omega$ as PSL $(2, q)$ in its usual doubly transitive representation, or

(b) $\Pi$ has order 9 and $M$ acts on $\Omega$ as $P S L(2,5)$ in its primitive representation of degree 10 .

Proof. We use the same notation of Lemma 4.7. We have that $M \cong$ $\operatorname{PSL}(2, q), q$ odd, by Theorem A. First, we shall prove that the subplane $\Sigma$ coincides with $\Pi$.

Suppose that $\Sigma \neq \Pi$. As we already showed in Lemma 4.7, the axes of homologies in $M$ lie in $\Sigma, \Omega$ and $\Sigma$ have no common points and each point of $\Omega$ belongs to the axis of exactly one homology in $M$.

Assume that $q \equiv 1(4), q \neq 9$. Let $\alpha, \beta$ be two involutions in $M$ which generate a dihedral group $D$ of order $q+1$. The axes of $\alpha$ and $\beta$ intersect in a point $P$ which lies in $\Sigma$ and is fixed by $D$. Since $D$ is a maximal subgroup of $M$-we suppose $q \neq 9$ - and $M$ is f.p.f. on $\Sigma$ by [10, Theorem 5.5], the point $P$ belongs to an orbit of length $|M| /|D|=q(q-1) / 2$. Furthermore, the centres of homologies in $M$ lie in an orbit of length $q(q+1) / 2$ contained in $\Sigma$ because the centralizer of an involution in $M$ is dihedral of order $q-1$. Thus, $\Sigma$ has at least $q^{2}$ points and hence $\Sigma$ has order $m \geq q$. On the other hand, it is easily seen that $n=q(q+1)-1$ and hence $m \leq q-1$ by Bruck's Theorem because $n$ is not a square. So, we have a contradiction.

Suppose that $q=9$. If $\Sigma$ contains a point $P$ such that $M_{P}>D$, where $D$ is dihedral of order 10 , then $M_{P} \cong P S L(2,5)$. So, $M_{P}$ contains a Klein group $K$ such that $\mathscr{N}_{M_{P}}(K) \cong A_{4}$. This is a contradiction. Indeed, $K$ is a triangular 
group of homologies and the vertices of $F(K)$ are cyclically permuted by $A_{4}$. Therefore, $A_{4}$ must be f.p.f. on $\Pi$.

When $q \equiv 3(4), q \neq 7,11$, we obtain a contradiction as in the case $q \equiv 1$ (4), $q \neq 9$. Here $D$ is dihedral of order $q-1$.

Lastly, if $q=7$ or 11 and $M_{P}>D$ for some point $P \in \Sigma$, then $M_{P} \cong S_{4}$ or $P S L(2,5)$, respectively. In both cases we obtain a contradiction as in the case $q=9$.

Therefore $G$ is strongly irreducible over $\Pi$.

Let $H$ be the subset of $M$ consisting of all involutory homologies in $M$. Since $M$ is normal in $G$ and $G$ is transitive on $\Omega$, each point of $\Omega$ must lie in the axis of some homology in $H$. All involutions in $H$ are conjugate under $M$. Furthermore, the axis $l$ of any homology $\sigma \in H$ is fixed by an element of $M$ which interchanges the points $P, Q \in L \cap \Omega$. Indeed, by Lemma 2.4, $C_{G}(\sigma)$ cannot fix $P$ since it contains a Klein group. Therefore, $M$ is transitive on the points of $\Omega$.

We notice that if a point $P \in \Omega$ lies in the axis of exactly one involutory homology $\sigma \in M$, then no involutory homology in $G$, different from $\sigma$, can have the axis through $P$. Indeed it would be forced to centralize $\sigma$, contrary to Lemma 2.4.

For any involutory homology $\sigma \in M$, we denote by $C(\sigma)$ a subgroup of $C_{M}(\sigma)$ such that $C(\sigma)$ contains no Klein groups and $\left[C_{M}(\sigma): C(\sigma)\right]=2$. If $l$ is the axis of $\sigma$ and $P \in l \cap \Omega$, then it is easily seen that the stabilizer of $P$ in $C_{M}(\sigma)$ is a group of type $C(\sigma)$.

If $q \equiv 3$ (4) and $\sigma_{1}, \sigma_{2}$ are two distinct involutory homologies in $M$ then $C\left(\sigma_{1}\right)$ and $C\left(\sigma_{2}\right)$ are cyclic groups of order $(q+1) / 2$. We have that either $\left\langle C\left(\sigma_{1}\right), C\left(\sigma_{2}\right)\right\rangle=M$ or $\left\langle C\left(\sigma_{1}\right), C\left(\sigma_{2}\right)\right\rangle \cong A_{4}, S_{4}$ or $A_{5}$. By Lemma 2.4 , the axes of $\sigma_{1}$ and $\sigma_{2}$ cannot intersect in a point of $\Omega$. So, we have (1) for $q \equiv 3$ (4).

Suppose that $q \equiv 1$ (4). Again, let $\sigma_{1}, \sigma_{2}$ be two distinct involutory homologies in $M$. In this case $C\left(\sigma_{1}\right)$ and $C\left(\sigma_{2}\right)$ are cyclic groups of order $(q-1) / 2$. One can prove that if $\left\langle C\left(\sigma_{1}\right), C\left(\sigma_{2}\right)\right\rangle$ is different from $M$ and contains no Klein groups, then either $\left\langle C\left(\sigma_{1}\right), C\left(\sigma_{2}\right)\right\rangle \cong \mathscr{N}_{G}(P)$ where $P$ is a Sylow $p$-subgroup of $M$ for $q=p^{h}$, or $\left\langle C\left(\sigma_{1}\right), C\left(\sigma_{2}\right)\right\rangle \cong S_{3}$ and $q=5$. In both cases, $\left\langle C\left(\sigma_{1}\right), C\left(\sigma_{2}\right)\right\rangle$ is a maximal subgroup of $M$. So, if the axes of $\sigma_{1}$ and $\sigma_{2}$ intersect in a point $Q$ of $\Omega$, then either $M_{Q} \cong \mathscr{N}_{G}(P)$ or $q=5$ and $M_{Q} \cong S_{3}$. As in [2, Main Theorem], we have (2).

Clearly, if there are exactly one homology axis through each point of $\Omega$, we have (1).

EXAMPLE 4.9. The case (1) of Theorem 4.8 actually occurs. 
Let $\Pi$ be the Desarguesian plane of order 29. Then $\Pi$ admits a collineation group $F \cong P S L(2,29)$ which preserves a conic $\Omega$ and acts on the points of $\Omega$ in its usual doubly transitive representation. $F$ possesses a subgroup $M \cong P S L(2,5)$. Let $P$ be any point of $\Omega$. Since $\left|F_{P}\right|=14 \cdot 29,|M|=60$ and $|\Omega|=30$, we have that $\left|M \cap F_{P}\right|=2$ and $M$ is transitive on $\Omega$. Clearly, each point of $\Omega$ contains the axis of exactly one homology in $M$ because $\left|M \cap F_{P}\right|=2$.

Proof of Theorem B. When $4 \nmid|G|$ we have (i). If $4 \| G \mid$ and $G$ is not irreducible, then we have (i) by Proposition 4.3(1.b), provided $\Pi$ has order different from 5 . When $\Pi$ has order 5 , we may have also $G \cong A_{4} \cong \operatorname{PSL}(2,3)$ by Proposition 4.3(2), and the case (ii) occurs.

When $4 \| G \mid$ and $G$ is irreducible we have (ii) or (iii) by Theorem 4.8. Notice that in the case (1) of Theorem 4.8, the group $M$ is minimal transitive on $\Omega$. Indeed, any transitive subgroup of $M$ must contain all involutory homologies in $M$ and hence it coincides with $M$. In case (2.a), a direct inspection of the subgroups of $P S L(2, q), q \equiv 1$ (4), shows that $M$ is minimal transitive on $\Omega$ for all $q \neq 5,9$ or 29 . For $q=5, P S L(2, q)$ contains the subgroup $A_{4}$ which is minimal transitive on $\Omega$. For $q=9$ or $29, \operatorname{PSL}(2, q)$ contains a subgroup isomorphic to $\operatorname{PSL}(2,5)$ which is minimal transitive on $\Omega$. This leads to the case (2.b) or to Example 4.9.

When $4 \nmid|G|$ and $G$ is transitive on $\Omega, G$ must contain only involutions which induce odd permutations on $\Omega$. In this case we have not been able to obtain any significant information about the structure and the action of $G$.

\section{References}

[1] M. Biliotti and G. Korchmaros, 'Collineation groups strongly irreducible on an oval', Ann. Discrete Math. 30 (1986), 85-97.

[2] M. Biliotti and G. Korchmaros, 'Collineation groups which are primitive on an oval of a projective plane of odd order', J. London Math. Soc. (2) 33 (1986), 525-534.

[3] M. Biliotti and G. Korchmaros, 'Hyperovals with a transitive collineation group', Geom. Dedicata 24 (1987), 269-281.

[4] F. Buekenhout, 'The geometry of finite simple groups', Buildings and the geometry of diagrams, (Lecture Notes in Mathematics, vol. 1181, Springer-Verlag, Berlin, Heidelberg, New York and Tokyo, 1986).

[5] P. Dembowski, Finite geometries, (Springer-Verlag, Berlin, Heidelberg and New York, 1968).

[6] B. Fischer, 'A characterization of the symmetric groups on 4 and 5 letters', J. Algebra 3 (1966), 88-98.

[7] G. Glauberman, 'Central elements in core-free groups', J. Algebra 4 (1966), 403-420.

[8] D. Gorenstein, Finite groups, (Harper \& Row, New York, 1968).

[9] D. Gorenstein, The classification of finite simple groups, (Plenum Press, New York and London, 1983). 
[10] C. Hering, 'On the structure of finite collineation groups of projective planes', Abh. Math. Sem. Univ. Hamburg 49 (1979), 155-182.

[11] B. Huppert, Endliche Gruppen I, (Springer-Verlag, Berlin, Heidelberg and New York, 1979).

[12] B. Huppert and N. Blackburn, Finite groups III, (Springer-Verlag, Berlin, Heidelberg and New York, 1982).

[13] W. M. Kantor, 'On the structure of collineation groups of finite projective planes', Proc. London Math. Soc. (3) 32 (1976), 385-402.

[14] H. H. Mitchell, 'Determination of the ordinary and modular linear groups', Trans. Amer. Math. Soc. 12 (1911), 207-242.

[15] A. Reifart and G. Stroth, 'On finite simple groups containing perspectivities', Geom. Dedicata 13 (1982), 7-46.

Dipartimento di Matematica

Università di Lecce

Via Arnesano

73100 Lecce

Italia
Istituto di Matematica Università della Basilicata

Via N. Sauro 34

85100 Potenza

Italia 